\title{
Linear Response Theory of Ion Excitation for Fourier Transform Mass Spectrometry
}

\author{
Shenheng Guan \\ Department of Pharmaceutical Chemistry, University of California, San Francisco, California, USA
}

\begin{abstract}
A linear relation between the voltage density or magnitude spectrum of an excitation waveform and the corresponding ion orbital radius is derived for Fourier transform mass spectrometry (FTMS). This provides a theoretical foundation for the stored waveform inverse Fourier transform excitation method. The result is also useful for the design of optimal excitation signals as well as for the estimation of the kinetic energy of ions after an excitation event in collision-induced dissociation experiments. When the linear relation is applied to two-dimensional FTMS excitation, an analytical expression for ion speed modulation is obtained. (J Am Soc Mass Spectrom 1991, 2, 483-486)
\end{abstract}

$\mathrm{F}$ ourier transform mass spectrometry (FTMS), also known as Fourier transform ion cyclotron resonance (FT-ICR), has become a very powerful technique for mass analysis and for ion-molecule reaction studies after having undergone 16 years of development [1]. A potential capability of the FTMS method is to perform multistage tandem mass spectrometry (MS/MS) [2] in a single-cell instrument. FTMS excitation methods play a central role in implementing MS/MS experiments. In a typical MS/MS experiment, parent ions of interest are isolated by an ejection pulse and are then accelerated to an orbit with desired kinetic energy by an excitation pulse for collision-induced dissociation. It is important to be able to produce selective waveforms for ion isolation and to control ion kinetic energy with excitation pulses. These capabilities can be provided by the stored waveform inverse Fourier transform (SWIFT) excitation method introduced by Marshal and co-workers [1d, 3-7].

The demonstrated advantages of the SWIFT excitation technique include high resolution excitation [3] and ejection [7] and an extension of the detection dynamic range [4]. The high resolution nature of SWIFT can be used to design nearly perfect "masks" for Hadamard transform experiments [8]. Many other advantages are still waiting for the experimental proof. SWIFT provides excitation with more uniform spectral magnitude and thus improves the accuracy of relative ion abundance measurements (as for determination of the isotope ratio).

Although the inverse Fourier transform excitation originated in nuclear magnetic resonance for solvent suppression [9], the SWIFT theory has been mainly

Address reprint requests to Shenheng Guan, Department of Pharmaceutical Chemistry, University of California, San Francisco, CA 94143 developed from FTMS [1d, 3-7, 10, 11]. The reason for this is probably that the trapped ICR systems are more linear. However, the SWIFT method can be applied to certain applications for magnetic resonance and laser spectroscopy. Under the small-angle approximation, the Bloch equations reduce to linear equations that have the same structure as the Lorentz equations.

The SWIFT method is also called "tailored" excitation, and the near-linear relation between magnitude spectra and the responding signal magnitude spectra has been experimentally demonstrated [5]. The response to excitation has recently been studied by a numerical method [12]. Understanding of the response of FTMS excitation has largely been based on the single-frequency or "burst" model [13, 14], which is invalid in cases of SWIFT or frequency sweeping excitation. The response to other common FTMS excitation waveforms was studied by Marshall and coworkers $[15,16]$. In this work, a linear relation between the voltage density and the corresponding ion radius is derived through following the linear response theorem on ion motion based on the assumption of a uniform excitation field. This relation is important for MS/MS experiments performed on FTMS equipment becausc the ion radius is closely connected to its kinetic energy. The linear relation between ion transverse velocity and excitation voltage spectrum is helpful for understanding multipulse experiments without explicitly solving the Lorentz equations. An example for application of the method in two-dimensional (2D) FTMS excitation is givert.

The theory developed in this article clearly provides a simple and fast procedure for analyzing FTMS excitation events by avoiding laborious work on ion trajectory calculations. In that the present work is based on the assumption of uniform excitation field, 
future research should expand the results to more general cases where nonuniform excitation frelds are applied.

\section{Ion Excitation and Linear Response}

Classically, the motion of a charged particle with mass of $m$ and charge of $q$ in a magnetic feld $B$ and an electric field $E$ near zero pressure and zero charge density can be described by the Lorentz equation

$$
\frac{\mathrm{d} v}{\mathrm{~d} t}=\frac{q}{m}(E+v \times B)
$$

where $v$ is the velocity of the ion. In most FTMS experiments, a static magnetic field is used and it can be assigned along the $z$-axis

$$
B=B_{0} \mathbf{k}
$$

where $B_{0}$ is the magnitude of the magnetic induction and $\mathbf{k}$ is the unit vector in the $z$-direction. In all Fourier transform mass spectrometers, ion-trapping cells are used. The trapping cell provides a static electric potential to prevent ions escaping from the cell. The electric field generated by the trapping potential is usually small compared with that from excitation signals near the center of the cell. A recently introduced screened cell almust completely eliminates the trapping field inside the cell [17]. In this article we simply omit the electrostatic field and its consequences (e.g., ICR frequency shift as a function of ICR orbital radius and trapping voltage, magnetron motion, and other nonlinear effects) [18]. The electric freld of the excitation signal is assumed to be applied along the $x$-axis,

$$
E=E_{x}(t) \mathbf{i}
$$

where $E_{x}(t)$ is the magnitude of the excitation electric field and $\mathbf{i}$ is the unit vector along the $x$-direction. By bringing eqs 2 and 3 into eq 1 and neglecting the motion in the $z$-direction, we have the component equations:

$$
\begin{gathered}
\frac{\mathrm{d} v_{x}}{\mathrm{~d} t}=\frac{q}{m} E_{x}(t)+\omega_{0} v_{y} \\
\frac{\mathrm{d} v_{y}}{\mathrm{~d} t}=-\omega_{0} v_{x} \\
\omega_{0}=\frac{B_{0} q}{m}
\end{gathered}
$$

We can combine eqs 4 and 5 by introducing a complex transverse velocity $w$

$$
\begin{gathered}
\frac{\mathrm{d} w}{\mathrm{~d} t}=-i \omega_{0} w+\frac{q}{m} E_{x}(t) \\
w=v_{x}+i v_{y}
\end{gathered}
$$

or equivalent

$$
\frac{\mathrm{d}}{\mathrm{d} t}\left[w(t) e^{i \omega_{0} t}\right]=\frac{q}{m} E_{x}(t) e^{i \omega_{0} t}
$$

In all FTMS experiments, ion excitation is carried out in a pulsed mode; that is, excitation events occupy limited time durations. We first consider the effect of a single pulse in which excitation starts at $t=t_{1}$ and terminates at $t=t_{2}$. Outside $\left[t_{1}, t_{2}\right]$, the amplitude of the pulse is zero. The purpose of the excitation is to generate a desirable final value of ion velocity at $t=t_{2}\left[w\left(t=t_{2}\right)\right]$ from a given initial velocity at $t=t_{1}$. Integration of eq 9 from $t=t_{1}$ to $t=t_{2}$ and substituting $\omega_{0}$ for $\omega$ give

$$
\begin{aligned}
& w\left(\omega, t=t_{2}\right) e^{i \omega t_{2}}-w\left(\omega, t=t_{1}\right) e^{i \omega t_{1}} \\
& =\int_{t_{1}}^{t_{2}} \frac{q}{m} E_{x}(\tau) e^{i \omega r} \mathrm{~d} \tau
\end{aligned}
$$

We have defined the excitation signal to be zero outside of $\left[t_{1}, t_{2}\right]$, so the integration of the above equation can be expanded to infinity in both directions. This implies that the initial and final states of the complex transverse velocity are related to the Fourier transform ${ }^{1}$ of the excitation electric field. If the distance between the two excitation plates of the cell is $d$, the electric field is proportional to the voltage between the plates $\left(E_{x}=V / d\right)$ under the approximation of infinitely large plates. It should be noted that FTMS experiments are carried out in ion traps in which ions experience more complicated excitation fields. The proper treatments for ion excitation in a cubic or cylindrical trap can be found in Rempel et al. [19] and van der Hart and van de Guchte [20]. Equation 10 can be rewritten as

$$
\begin{gathered}
w\left(\omega, t_{2}\right) e^{i \omega t_{2}}-w\left(\omega, t_{1}\right) e^{i \omega t_{1}}=\frac{q}{m d} V(\omega) . \\
V(\omega)=F(\omega) e^{i P(\omega)}
\end{gathered}
$$

Here $V(\omega)$ is the complex voltage spectrum of the excitation signal, which is the Fourier transform of the time-domain voltage signal, $F(\omega)$ is the voltage density or voltage magnitude spectrum, and $P(\omega)$ is its phase spectrum.

Thus far the most general relation between the voltage spectrum of excitation and the ion velocities has been derived. 'This result can be used for analysis of multipulse experiments. An example of the applica-

\footnotetext{
${ }^{1}$ For the convention of Fourier transformation, the sign in the exponential of eq 10 shoutd be negative. The equation shows a positive sign, which reflects the fact that the direction of the angular velocity $(\omega)$ is opposite to the direction of the applied static magnetic field for a positive charge. This results in the negative cyclotron frequency.
} 
tion of the general equation (eq 11) will be given in the next section. However, we shall now return to the simplest experiment, which contains only one excitation pulse. Suppose the initial velocity is zero $\left[w\left(t_{1}\right)\right.$ $=0]$ and the excitation terminates at $t=0\left(t_{2}=0\right)$. Equation 11 becomes

$$
w(\omega)=\frac{q}{m d} V(\omega)
$$

This implies that the final velocity of ions is proportional to the voltage density of the excitation pulse. The proportionality between the voltage density of excitation and the responding ion velocity shows clear theoretical evidence that FTMS excitation belongs to linear responding systems with the assumption of uniform excitation field. The left side of the equation also relates to the radius $(r)$ of the ion

$$
r(\omega)=\frac{|w(\omega)|}{\omega}=\frac{F(\omega)}{B_{0} d}
$$

or

$$
F(\omega)=\left(B_{0} d\right) r(\omega)
$$

This equation is important in the synthesis of the excitation signal. One can achieve the desired radii for any mass-to-charge ratio or cyclotron frequency (eq 6) by specifying the corresponding voltage density of the excitation waveform. These results can be used for prediction of the resultant ion signals $[13,19,21]$. The kinetic energy $\left(E_{k}\right)$ of the ion can also be correlated to the voltage density

$$
E_{k}(\omega)=\frac{1}{2} m|w(\omega)|^{2}=\frac{q^{2}}{2 m d^{2}} F^{2}(\omega)
$$

If the initial ion velocities are assumed to be zero, these equations imply that for single-pulse experiments the ion radius and kinetic energy do not depend on the phase spectrum $[P(\omega)]$ of the excitation waveform. Therefore, the magnitude spectrum of the responding ion signals is also independent of the phase. In contrast, voltage density alone cannot determine the time-domain waveform (excitation signal) uniquely. The corresponding phase function has to be specified. In a prior work [10], a general method for producing optimal phase functions has been described. Phase functions of excitation pulses have no physical significance in those single-pulse experiments, so they can be chosen for dynamic reduction.

\section{Speed Modulation from Two- Dimensional Excitation}

For multipulse experiments, phase relation among excitation pulses plays a key role. In a previous work [22], a theoretical model for 2D FTMS was presented; the postexcitation ionic speed modulation was derived for two identical single-frequency excitation pulses separated by a variable delay that represents the time scale for the second dimension. In this section, the general equation (eq 11) developed in the last section is applied to 2D excitation.

Two-dimensional FTMS signals come from speed modulation which is produced by $2 \mathrm{D}$ excitation consisting of a pair of excitation pulses (Figure 1). At $t=0$, the initial ion speed is assumed to be zero. According to eq 11 , the transverse velocity of ions at the end of the first pulse is

$$
w(T) e^{i \omega T}=\frac{q}{m d} V(\omega)
$$

where $V(\omega)$ is the complex voltage spectrum of the first excitation pulse. We still can use eq 11 to derive the transverse velocity after the evolution period $(t=$ $\left.T+t_{1}\right)$ by setting $V(\omega)$ equal to zero for $T<t<T+$ $t_{1}$. This gives

$$
w\left(T+t_{1}\right) e^{i \omega\left(T+t_{1}\right)}-w(T) e^{i \omega T}=0
$$

or

$$
w\left(T+t_{1}\right) e^{i \omega\left(T+t_{1}\right)}=w(T) e^{i \omega T}=\frac{q}{m d} V(\omega)
$$

The complex voltage spectrum of the second pulse can be expressed as $V(\omega) e^{i \omega\left(T+t_{1}\right)}$ because it is timeshifted from the origin with a distance of $T+t_{1}$. Using eq 11 once again during $\left[T+t_{1}, 2 T+t_{1}\right]$, we have

$$
\begin{aligned}
& w\left(2 T+t_{1}\right) e^{i \omega\left(2 T+t_{1}\right)}-w\left(T+t_{1}\right) e^{i \omega\left(T+t_{1}\right)} \\
& =\frac{q}{m d} V(\omega) e^{i \omega\left(T+t_{1}\right)}
\end{aligned}
$$

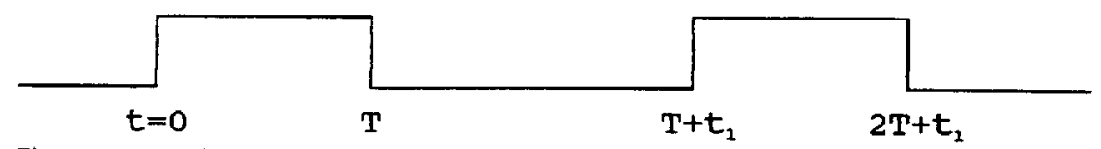

Figure 1. Two-dimensional FTMS excitation. The two identical pulses are used to produce speed modulation necessary for $2 \mathrm{D}$ experiments. The second pulse can be considered as time-shifting of the first pulse with a shifting distance of $T+t_{1}$. If the complex voltage spectrum of the first pulse is $V(\omega)$, the second pulse has a voltage spectrum of $V(\omega) e^{i\left(T+t_{1}\right)}$. 
Substituting eq 19 into the above, one can obtain

$$
w\left(2 T+t_{1}\right)=\frac{q}{m d} V(\omega) e^{-i \omega\left(2 T+t_{1}\right)}\left(1+e^{i \omega\left(T+t_{1}\right)}\right)
$$

The speed modulation is therefore

$$
\left|w\left(2 T+t_{1}\right)\right|=\frac{q}{m d}|V(\omega)|\left\{2\left[1+\cos \omega\left(T+t_{1}\right)\right]\right\}^{1 / 2}
$$

Because the pulse length $T$ is a constant in the 2D experiment, the ion speed at the end of the second pulse is modulated with $\omega t_{1}$. The above equation is not limited to single-frequency pulses and is more general than that derived in the previous work (see eq 2 in Guan and Jones [22]). From this result, one can also conclude that speed modulation of $2 \mathrm{D}$ excitation is independent of the phase functions of individual pulses. Therefore arbitrary excitation pulses can be used for 2D excitation purposes.

\section{Conclusion}

We have derived a linear relation between voltage density or magnitude spectrum of an FTMS excitation waveform and its corresponding ion radius based on the assumption of uniform excitation field. For a SWIFT excitation one can first specify ion radius as a function of frequency (or "radius spectrum") to achieve desired excitation. In other cases, the timedomain waveforms are usually defined first. The relation allows one to estimate the final ion kinetic energy gained from an excitation process. The linear relation provides a convenient tool for analysis of multipulse experiments. This is demonstrated for the case of $2 \mathrm{D}$ FTMS excitation.

\section{References}

1. Reviews of this field include the following: (a) Burlingame, A. L.; Millington, D. S.; Norwood, D. L.; Russell, D. H Anal. Chem. 1990, 62, 268R. (b) Freiser, B. S. In Techniques for the Study of Ion Molecule Reactions; Farrar, J. M.; Saun- ders, M. Jr., Eds.; Wiley: New York, 1988. (c) Gross, M. L.; Rempel, D. L. Science (Washington, DC) 1984, 226, 261-268. (d) Marshall, A. G.; Wang, T.-C. L.; Chen, L.; Ricca, T. L. In Fourier Transform Mass Spectrometry: Evolution, Innovation, and Applications; Buchanan, M. V., Ed; ACS Symposium Series 359; American Chemical Society, Washington, DC, 1987; pp 21-33. (e) Nibbering, N. M. M. Acc. Chem. Res. 1990, 23, 279. (f) Russell, D. H. Mass Spectrom. Rev. 1986, 5 , 167-189. (g) Wilkins, C. L. Mass Spectrom. Rev. 1988, 8, 67-92. (h) Wanczek, K.-P. Int. J. Mass Spectrom. Ion Processes 1989, 95, 1 .

2. Cody, R. B.; Freiser, B. S. Int. J. Mass Spectrom. Ion Phys. 1982. 41, 199. McIver, R. T.; Bowers, W. D. In Tandem Mass Spectrometry; Mçafferty, F. W., Ed.; Wiley: New York, 1983; p 287.

3. Marshall, A. G.; Wang, T.-C. L.; Ricca, T. L. I. Am. Chem. Soc. $1985,107,7893$.

4. Wang, T.-C. L.; Ricca, T. L.; Marshall, A. G. Anal. Chem. 1986, $58,2935$.

5. Chen, L.; Wang, T.-C. L.; Ricca, T. L.; Marshall, A. G. Anal. Chem. 1987, 59, 449.

6. Chen, L.; Marshall, A. G. Rapid Commun. Mass Spectrom. 1987, 1, 39.

7. Chen. L.; Marshall, A. G. Int. I. Mass Spectrom. Ion Processes 1987, 79, 115.

8. Williams, E. R.; Loh, S. Y.; McLafferty, F. W.; Cody, R. B. Anal. Chem. 1990, 62, 698.

9. Tomlinson, B. L.; Hill, H. D. W. J. Chem. Phys. 1973, 59, 1775.

10. Guan, S.; Mclver, R. T., Jr. J. Chem. Phys. 1990, 92, 5841.

11. Guan, S. J. Chem, Phys, 1989, 91, 775.

12. Hearn, B. A.; Watson, C. H.; Baykut, G.; Eyler, J. R. Int. J. Mass Spectrom. Ion Processes 1990, 95, 299.

13. Comisarow, M. B. J. Chem. Phys. 1978, 69, 4097.

14. Goodman, S. U.S. Patent $4945234,1990$.

15. Wang, M. D.; Marshall, A. G. Int. J. Mass Spectrom. Ion Processes 1990, 100, 323.

16. Grosshans, P. B.; Marshall, A. G. Int. I. Mass Spectrom. Ion Processes 1990, 100, 381.

17. Wang, M. D.; Marshall, A. G. Anal. Chem. 1990, 62, 515.

18. Rempel, D. L.; Grese, R. P.; Gross, M. L. Int. I. Mass Spectrom. Ion Processes 1990, 100, 381.

19. Rempel, D. L.; I luang, S. K.; Gross, M. L. Int. I. Mass Spectrom. Ion Processes 1986, 70, 163.

20. van der Hart, W. J.; van de Guchte, W. J. Int. J. Mass Spectrom. Ion Processes 1988, 82, 17.

21. Dunbar, R. C. Int. J. Mass Spectrom. Ion Processes 1984, 70, 163.

22. Guan, S.; Jones, P. R. J. Chem. Phys. 1989, 91, 5291. 